\title{
FORUM
}

\section{EUFOR Tchad/RCA: Zwischenbilanz und Empfehlungen für eine effektivere Konfliktlösung}

\author{
Hans-Georg Ehrhart*
}

Seit 2003 finden im Krisendreieck Darfur-Tschad-Zentralafrikanische Republik ein Stellvertreterkrieg und eine humanitäre Tragödie statt, deren Hauptprotagonisten die Regierungen des Sudan und des Tschad sind. In der Region schien sich im Laufe des Jahres 2007 ein ,Fenster der Möglichkeiten` zu öffnen: Im Juli 2007 wurde die gemeinsame Operation (,Hybridmission") von UN und Afrikanischer Union (AU) für Darfur (UNAMID) ${ }^{1}$ beschlossen und zwei Monate später die multi-dimensionale UN-Mission im Tschad und in der Zentralafrikanischen Republik (MINURCAT). ${ }^{2}$ Im Tschad begann im Februar 2007 ein interner politischer Dialog, der zu Wahlen im Jahr 2009 führen sollte. Ende Oktober 2007 fand in Libyen eine von der UN und der AU organisierte Friedenskonferenz über Darfur statt, die in einen graduellen Friedensprozess münden sollte. Beide Prozesse sind jedoch extrem gefährdet.

Am 24. September 2008 verlängerte der Sicherheitsrat der Vereinten Nationen einstimmig das Mandat der MINURCAT um ein halbes Jahr. ${ }^{3}$ Sie war ein Jahr zuvor beschlossen worden mit dem Ziel, ein sicheres Umfeld zu schaffen und die freiwillige Rückkehr der 290.000 Flüchtlinge aus Darfur und der Zentralafrikanischen Republik (RCA) sowie der 180.000 Vertriebenen innerhalb des Tschad zu ermöglichen. ${ }^{4}$ EUFOR Tchad/RCA sollte für ein Jahr die militärische Komponente eines komplexen Unternehmens stellen, das aus drei Komponenten besteht:

- einem zivilen UN-Element, das für die Ausbildung der tschadischen Polizei, Menschenrechtsschutz und die Förderung der Rechtsstaatlichkeit zuständig ist;

- einem auszubildenden Kontingent der tschadischen Polizei, das für den Schutz der über 400.000 Flüchtlinge und Vertriebenen in den Lagern sorgen soll;

- und einer von der Europäischen Union gestellten militärischen Komponente, EUFOR Tchad/RCA, welche die MINURCAT unterstützen soll. ${ }^{5}$

Der Auftrag dieser bislang größten autonomen Militäroperation der Europäischen Union besteht darin, im Osten des Tschad und im Nordosten der Zentralafrikanischen Republik zu einem sicheren Umfeld beizutragen und so die Voraussetzungen für eine Rückkehr der

* Dr. Hans-Georg Ehrhart, Leiter des Zentrums für Europäische Friedens- und Sicherheitsstudien am Institut für Friedensforschung und Sicherheitspolitik an der Universität Hamburg.

1 African Union/United Nations Hybrid Mission in Darfur.

2 MINURCAT soll 300 Polizisten, 50 Verbindungsoffiziere und eine angemessene Zahl von zivilem Personal umfassen. Vgl. UN-Sicherheitsrat: Resolution 1778 (2007), adopted by the Security Council at its 5748th meeting, on 25 September 2007, S/RES/1778 (2007), vom 25. September 2007, S. 3.

3 UN-Sicherheitsrat: Resolution 1834 (2008), adopted by the Security Council at its 5981st meeting, on 24 September 2008, S/RES/1834 (2008), vom 24. September 2008.

4 Vgl. Internetseite der MINURCAT, abrufbar unter: http://www.un.org/Depts/dpko/missions/minurcat/ (letzter Zugriff: 10.12.2008)

5 Vgl. zur Vorgeschichte Hans-Georg Ehrhart: EU-Krisenmanagement in Afrika: die Operation EUFOR Tchad/ RCA, in: integration 2/2008, S. 145-158. 
Flüchtlinge in ihre Heimat zu verbessern. EUFOR ist als Brückenmission konzipiert, das heißt ihre Präsenz endet am 15. März 2009, also ein Jahr nach Herstellung der Einsatzbereitschaft. Ihr soll eine Militärmission der UN nachfolgen. Die Planungen für diese Nachfolgeoperation laufen bereits. Im Dezember 2008 legte der UN-Generalsekretär einen Bericht über Größe, Struktur und Mandat vor und anschließend wird der Sicherheitsrat endgültig über die neue Mission entscheiden. ${ }^{6}$ So weit der politische Fahrplan. Aber wie ist der bisherige Einsatz von EUFOR zu bewerten? Wie sind die Aussichten für eine möglichst reibungsfreie Übernahme des militärischen Sicherungsauftrages durch die UN? Wie könnten Schritte zu einer Konfliktlösung aussehen? Was sollte die Europäische Union dabei tun?

\section{Gemischte Bilanz}

Der quälend langsame Beginn der EUFOR-Operation gab nicht unbedingt Anlass zu großem Optimismus. Es dauerte immerhin sechs Monate bis zur Herstellung der vorläufigen Einsatzbereitschaft. Die im Krisenmanagementkonzept genannte Zahl der Einsatzkräfte musste zudem von 4.000 auf 3.700 reduziert werden, weil auf den fünf Truppenstellerkonferenzen nicht genug Zusagen kamen. Bis Mitte Oktober 2008 waren erst 3.300 am Einsatzort. Das größte EUFOR-Kontingent stellt Frankreich mit 1.700 Soldaten, gefolgt von Irland und Polen mit je gut 400 Einsatzkräften. Während Österreich, das zu der Zeit einen Sitz im Weltsicherheitsrat anstrebte, mehr als 160 Soldaten stellt, ist Deutschland nur mit vier Offizieren im Hauptquartier vertreten. Insgesamt beteiligen sich 15 Nationen mit Truppen am Einsatzort, 25 sind im Hauptquartier Mont Valérien bei Paris vertreten. ${ }^{7}$

Die Einsatzkräfte am Ort müssen in einem extrem schwierigen Umfeld agieren. Dazu gehören die Größe des Einsatzgebietes, die völlig unterentwickelte Infrastruktur und das extreme Klima. Sie stellen hohe Anforderungen an Luftbeweglichkeit, Logistik und die Versorgung der eigenen Truppe mit Wasser. So besteht ein notorischer Mangel an Hubschraubern mit spezifischen Fähigkeiten. Diese Lücke konnte mittlerweile verkleinert werden, nachdem es im November 2008 nach langwierigen Verhandlungen gelang, von Moskau die Zusage über die Teilnahme von vier Mi-8-Helikoptern und 120 Soldaten zu erlangen. Damit nimmt Russland erstmals an einer Operation im Rahmen der Europäischen Sicherheits- und Verteidigungspolitik (ESVP) teil. ${ }^{8}$

Aber auch die politische Lage in der zentralafrikanischen Konfliktregion entwickelte sich nicht wie erhofft. Die Stationierung der von der AU und der UN gestellten Hybridmission UNAMID in Darfur kommt vor allem wegen der sudanesischen Obstruktionspolitik nicht voran. EUFOR sollte aber das stabilisierende Gegenstück zur UNAMID sein. Der über bewaffnete Milizen ausgetragene Konflikt zwischen Sudan und Tschad droht immer wieder aufzuflammen, auch wenn beide Staaten im November 2008 die diplomatischen Beziehungen wieder aufgenommen haben. Innerhalb des Tschad kommt der politische Versöhnungsprozess nicht voran, was nach der Ermordung eines Oppositionsführers durch die Präsidentengarde von Präsident Déby nicht erstaunlich ist. Zudem zeigt der wegen des tschadischen

6 UN-Sicherheitsrat: Report of the Secretary-General on the United Nations Mission in the Central African Republic and Chad, S/2008/760, vom 4. Dezember 2008. Ursprünglich sollte der Bericht bereits am 15. November 2008 vorliegen. Die für den 15. Dezember 2008 vorgesehene Mandatierung durch den UN-Sicherheitsrat wird erst im Januar 2009 erfolgen.

7 Mit Albanien, Kroatien und Russland sind drei Nicht-EU-Staaten beteiligt. Vgl. Übersicht: Force Strength by Nations in AOO: 3396, abrufbar unter: http://www.consilium.europa.eu/uedocs/cmsUpload/081117\%20Force $\% 20$ Stenght $\% 20$ by\%20Nations\%20in\%20AOO.pdf (letzter Zugriff: 10.12.2008).

8 Rat der Europäischen Union: Javier Solana, EU High Representative for the CFSP, signed an agreement with the Russian Ambassador to the EU on the participation of the Russian Federation in the operation EUFOR TCHAD/RCA, Pressemitteilung S357/08, vom 5. November 2008. 
Rohstoffreichtums von China und den USA hofierte Déby keine allzu große Kooperationsbereitschaft mit der UN.

Vor dem Hintergrund dieser Schwierigkeiten fällt die Einschätzung des bisherigen EUFOR-Einsatzes und seiner Einbindung in die mehrdimensionale MINURCAT-Mission gemischt aus. Der Hohe Repräsentant für die Gemeinsame Außen- und Sicherheitspolitik der Europäischen Union, Javier Solana, lobte in New York die Aktivitäten der EUFOR und ihre Zusammenarbeit mit der UN-Mission, räumte aber auch ein, dass die Sicherheitslage noch immer volatil sei. ${ }^{9}$ Diese Einschätzung wird grundsätzlich durch Berichte der UN ${ }^{10}$ und der Nichtregierungsorganisation „Oxfam“, die über langjährige Erfahrung im Tschad verfügt, geteilt. ${ }^{11}$ Gleichzeitig weisen UN- und NGO-Beobachter auf folgende Kritikpunkte hin:

Die Sicherheit der Flüchtlinge ist immer noch äußerst prekär. So erlaubt es das Mandat der EUFOR nicht, in den Lagern selbst für Sicherheit zu sorgen und polizeiartige Aufgaben zu übernehmen. Da die Polizeikräfte des Tschad nicht in der Lage sind Sicherheit zu gewährleisten, entsteht immer wieder ein Sicherheitsvakuum, das durch gewalttätige Kräfte - Banditen, Milizen, Kriminelle - ausgenutzt wird. Aber auch außerhalb der Lager bleibt die Sicherheitslage schwierig.

Auch die Helfer sind gefährdet. 2008 sind sechs humanitäre Helfer getötet und über 120 Angriffe gegen sie durchgeführt worden, sodass sie nur eingeschränkt handlungsfähig sind.

Ein weiterer Kritikpunkt ist die Tatsache, dass eine 3.300 Soldaten umfassende Truppe keinen Einsatzraum von über $300.000 \mathrm{~km}^{2}$ vollständig absichern kann.

Während ein Teil der Flüchtlinge die Präsenz der EUFOR positiv beurteilt, wissen viele nicht, was deren Aufgabe ist und dass sie sich von den französischen Truppen im Tschad unterscheidet. Es bedürfte also einer umfassenden Informationskampagne über Sinn und Zweck des EUFOR-Einsatzes.

Kritisch beurteilt wird die Nutzung weißer Fahrzeuge und Helikopter durch EUFORKräfte, denn sie vermindert die Unterscheidbarkeit von militärischen Kräften und humanitären Helfern.

Die zentrale Kritik richtet sich aber gegen die kurzfristige Perspektive, mit der diese Mission durchgeführt wird. Sie hat mit dazu beigetragen, dass die Rückkehr der Flüchtlinge, ein Hauptziel des ganzen Unternehmens, bislang nicht erreicht wurde. Andere Ursachen dafür liegen in der immer noch instabilen Sicherheitslage, die sich nach dem Ende der Regenzeit noch weiter verschlechtern dürfte, sowie in der Vernachlässigung des politischen Prozesses innerhalb des Tschad und der ganzen Krisenregion. Dies ist aber eine Aufgabe, die EUFOR nicht schultern kann. Gefordert sind vor allem der Tschad selbst, die anderen regionalen Akteure und nicht zuletzt die internationale Gemeinschaft.

\section{Schwieriger Stabwechsel}

Die UN ist mit der Herausforderung konfrontiert, eine Nachfolgemission für die EUFOR vorzubereiten. Die Grundzüge des vorläufigen Operationskonzepts sahen ursprünglich eine Infanteriebrigade mit ungefähr 6.000 Soldaten für ein vergrößertes Operationsgebiet im Tschad vor. Nach zähen Verhandlungen mit Präsident Déby einigte man sich schließlich auf

9 Rat der Europäischen Union: Intervention de Javier Solana, Haut Représentant de l'Union européenne pour la PESC, à l'occasion du débat sur EUFOR Tchad/RCA au Conseil de sécurité des Nations Unies, Pressemitteilung S317/08, vom 24. September 2008.

10 Vgl. UN-Sicherheitsrat: Report of the Secretary-General on the United Nations Mission in the Central African Republic and Chad, S/2008/444, vom 8. Juli 2008.

11 Vgl. Oxfam International: Mission incomplete: why civilians remain at risk in eastern Chad, Oxfam Briefing Paper 119/2008. 
eine Stärke von 4.900 Soldaten. ${ }^{12}$ Die Truppe wird also wesentlich größer sein als EUFOR und soll mit 18 Hubschraubern flexibel einsatzfähig sein. Allerdings hat die Europäische Union bereits sechs Monate gebraucht, um 16 Helikopter für EUFOR zur Verfügung zu haben. Zusätzlich ist eine „over the horizon“-Truppe in Bataillonsstärke vorgesehen, womit die Gesamtstärke auf 6.000 bis 6.500 Blauhelme steigen würde. Die Einsatzkräfte für die Zentralafrikanische Republik kämen noch hinzu. ${ }^{13}$

Das erste Problem lag darin, dass der Tschad ursprünglich nur eine wesentlich kleinere Blauhelmmission von 3.000 Einsatzkräften akzeptieren wollte. ${ }^{14}$ Nachdem er schließlich einem um 1.100 verringerten Ansatz zugestimmt hat, besteht die nächste Schwierigkeit darin, die geforderte Truppenstärke zu erreichen, denn gut ausgebildete Soldaten sind aufgrund des oftmals schwach ausgeprägten politischen Willens der Mitgliedstaaten Mangelware. ${ }^{15}$ Das Gleiche gilt für die hochwertige Ausrüstung für Luftbeweglichkeit und Aufklärungsfähigkeit. Diese ist aufgrund der unwirtlichen Lebensbedingungen, die alleine wegen des hohen Wasserbedarfs eine noch umfangreichere Personalstärke unmöglich machen, und der Größe des Operationsgebietes notwendig. Schwierig erscheint auch die zeitgerechte Übergabe von EUFOR auf die UN-Nachfolgetruppe. Existierende Unterkünfte müssen rechtzeitig ausgebaut und neue in Regionen, in denen EUFOR nicht präsent war, aufgebaut werden, sodass die Blauhelme gleich einsatzbereit sind. Zuallererst müssten also, noch vor Beginn der Rückführung von EUFOR, Unterstützungskräfte stationiert werden. Dazu gehören Luftunterstützung, Logistik und Ingenieurkräfte. Insgesamt alles knappe Ressourcen, die relativ kurzfristig von den UN-Mitgliedstaaten zur Verfügung gestellt werden müssten. Ein ,re-hatting' entsprechender EUFORKräfte wäre aus UN-Sicht wohl der beste Weg. Schließlich muss geklärt werden, ob die Blauhelme die von EUFOR genutzte Infrastruktur übernehmen dürfen. Diese Frage birgt problematische rechtliche und finanzielle Aspekte, über die gegenwärtig in New York verhandelt wird.

Insgesamt ist es ungewiss, ob die internationale Gemeinschaft das Konzept mit entsprechenden finanziellen, materiellen und personellen Beiträgen unterstützen wird. Dass die UN selbst Zweifel daran hegt zeigt zweierlei: Erstens schlägt der Generalsekretär vor, die Resolution für die Stationierung der UN-Truppe so lange als Entwurf zu belassen, bis genügend Zusagen eingegangen sind. Zweitens regt er an darüber nachzudenken, ob EUFOR im Falle einer verzögerten Stationierung die Lücke füllt. Die Europäische Union und die Bundesregierung sollten sich schon einmal auf eine entsprechende Anfrage vorbereiten.

\section{Schritte zur Konfliktlösung}

UN-Generalsekretär Ban Ki-moon hat in seinem Zwischenbericht von Juli 2008 kritisch darauf hingewiesen, dass die Mandate von MINURCAT und EUFOR bislang nur die Folgen, aber nicht die Ursachen des Konflikts im Tschad behandeln. Wenn die eigentlichen Ursachen aber nicht angegangen würden, dann liefe die internationale Gemeinschaft Gefahr, die investierten Ressourcen zu vergeuden. ${ }^{16}$ Immerhin handelt es sich mit 315 Millionen US-Dollar für MINURCAT und circa 700 Millionen Euro für EUFOR um insgesamt fast eine Milliarde Euro pro Jahr. Folglich darf es ein einfaches ,Weiter so!' eigentlich nicht ge-

12 Vgl. UN-Sicherheitsrat: Report of the Secretary-General, 4. Dezember 2008.

13 Für die RCA nennt der Bericht drei Optionen: 15 Militärbeobachter, ein Kontingent von 500 oder eines von 1.000 Soldaten.

14 Vgl. Security Council Report: Chad/CAR, Dezember 2008, abrufbar unter: http://www.securitycouncilreport.org/site/pp.aspx?c=glKWLeMTIsG\&b=4780487\&printmode=1 (letzter Zugriff: 10.12.2008).

15 Diese Knappheit ist durch den UN-Beschluss, weitere 3.000 Blauhelme in den Kongo zu entsenden, verschärft worden.

16 Vgl. UN-Sicherheitsrat: Report of the Secretary-General, 8. Juli 2008. 
ben. Trotzdem ist nicht auszuschließen, dass sich die Debatte in den kommenden Monaten einmal mehr um Fragen wie Truppenstationierung und Ausrüstung dreht und damit von der aktiven Auseinandersetzung mit den drei zentralen Konfliktursachen im Tschad ablenken wird: dem Staatsversagen, dem politischen Machtkampf und dem mittels Rebellengruppen geführten tschadisch-sudanesischen Stellvertreterkrieg.

Der Tschad ist im Grunde genommen ein ,schwacher' Staat, der sich an der Grenze des Scheiterns befindet. Die seit Kurzem fließenden Öleinnahmen haben die Lage nicht verbessert, weil sich Déby ihrer bedient, um seine Herrschaft durch einen Mix aus Unterdrückung und Vetternwirtschaft zu sichern statt Reformen voranzutreiben. Er hat in der Vergangenheit die These belegt, dass ein scheiternder Staat beziehungsweise die ihn regierende politische Gruppe durchaus ,erfolgreich`sein kann, solange ausreichend Renten generiert werden, um Unzufriedene, die nicht durch Unterdrückung ausgeschaltet werden können, zufrieden zu stellen. Statt sich um die Versorgung und die Entwicklung des ganzen Landes zu kümmern, überlässt er die Peripherie weitgehend sich selbst und der internationalen Staatengemeinschaft, welche die Kosten für das Staatsversagen zu tragen hat. ${ }^{17}$

Die geplante Folgemission der UN dürfte allerdings kaum in der Lage sein, den Staatsaufbau wirklich voranzubringen. Es ist zwar vorgesehen, mehr Polizisten auszubilden, die für die Sicherheit in den Flüchtlingslagern sorgen sollen, doch gehen damit noch nicht die institutionellen Reformen einher, die notwendig sind, um die Sicherheitskräfte adäquat zu führen. Erforderlich wäre ein langfristig ausgerichteter Prozess der Sicherheitssektorreform, der den Aspekt der demokratischen Kontrolle einschließt. Es ist positiv zu bewerten, dass Ban Ki-moon in seinem Bericht darauf drängt, das Mandat der künftigen MINURCAT dahin gehend zu erweitern, dass sie einen Beitrag zur Regelung der oben genannten drei Hauptprobleme erbringen kann. Zu befürchten ist aber, dass die Kooperationsbereitschaft von Déby mit der Stationierung der Nachfolgemission nicht gerade steigen wird. Sie nähme ihm Sicherheitslasten und sorgte für die Flüchtlinge ohne politische Gegenleistung dafür von ihm zu verlangen. ${ }^{18} \mathrm{Ob}-$ wohl der Zeitdruck für Déby arbeitet, sollte die Europäische Union ihre Tschad-Politik stärker politisieren und in eine internationale Strategie einbinden, die drei Aktionsfelder umfasst: ${ }^{19}$

Im ersten Aktionsfeld geht es darum, den politischen Prozess zwischen der Regierung einerseits und der gewaltfreien politischen Opposition sowie der tschadischen Zivilgesellschaft andererseits zu beleben, auszubauen und zu intensivieren. Nur durch einen inklusiven Dialog über Frieden, Sicherheit und Entwicklung kann eine Stabilisierung des Landes gelingen. Ziel sollte eine nationale Übereinkunft sein, in der strittige politische und wirtschaftliche Fragen wie Reform des politischen Systems, Sicherheitssektorreform, Justizreform, Dezentralisierung und Ressourcenzugang geregelt werden.

Das zweite Aktionsfeld beinhaltet den Aufbau und Ausbau eines Dialogprozesses mit der bewaffneten Opposition. Ziel wäre zunächst ein stabiler Waffenstillstand, ein gemeinsamer Überprüfungsmechanismus und ein Prozess der Entmilitarisierung, Demobilisierung und Reintegration. Im Rahmen einer umfassenden Reform des gesamten Sicherheitssektors könnte ein Teil der Rebellen in die Sicherheitskräfte integriert werden, während andere sich als politische Kraft etablieren und im ersten Aktionsfeld teilnehmen würden.

17 Vgl. dazu Annette Weber: Kriege ohne Grenzen und das ,erfolgreiche Scheitern“ der Staaten am Horn von Afrika, SWP-Studie 26/2008.

18 Vgl. Bjoern H. Seibert: Chad, Making sense of Darfur, Peacekeeping: UN/EU Midterm Review on Chad - A few thoughts, in: The Social Science Research Council Blogs, 25.09.2008, abrufbar unter: http://www.ssrc.org/blogs/ darfur/2008/09/25/uneu-midterm-review-on-chad-\%E2\%80\%93-a-few-thoughts/ (letzter Zugriff: 10.12.2008).

19 Vgl. dazu ausführlich International Crisis Group: Chad: A New Conflict Resolution Framework, Africa Report $144 / 2008$. 
Im dritten Aktionsfeld steht die regionale Dimension des Konflikts im Mittelpunkt. Diese bedarf eines international und vor allem durch die AU gestützten Mechanismus zur Konfliktlösung, der alle relevanten Akteure einschließt und zur Vertrauensbildung beiträgt. Seine wichtigsten Aufgaben wären dafür Sorge zu tragen, die grenzüberschreitenden Aktivitäten von Rebellengruppen zu unterbinden, die Sicherheit der im Grenzgebiet lebenden Menschen zu verbessern und den Waffenhandel zu bekämpfen.

\section{Rolle der Europäischen Union - Empfehlungen}

Die Europäische Union sollte diese umfassende Strategie unterstützen, indem sie zusammen mit der UN und afrikanischen Partnern Druck auf die politischen Akteure im Tschad in Form von positiven und negativen Anreizen ausübt. Sie muss sich diplomatisch, wirtschaftsund entwicklungspolitisch stärker engagieren, der AU und subregionalen Organisationen aber den Vorrang lassen. Konkret sollte sie ihre entwicklungspolitischen Aktivitäten, insbesondere im Bereich der politischen Reformen und der Stärkung der Zivilgesellschaft, beschleunigt umsetzen und intensivieren. Waffenlieferungen an die Regierung des Tschad durch EU-Mitgliedstaaten sollten unterlassen und Maßnahmen zur Unterbindung von Waffenlieferungen an Rebellengruppen unterstützt werden. Kurzfristig sollte EUFOR ihre Aktivitäten zum Schutz der Flüchtlinge und Vertriebenen verstärken. Der Übergang zu einer Nachfolgemission sollte erleichtert werden, indem EU-Mitgliedstaaten ihre Kontingente der UN unterstellen oder das EUFOR-Mandat verlängert wird, bis die vorläufige Einsatzbereitschaft der Blauhelme hergestellt ist. Die zweite Option ist sehr unwahrscheinlich, für die erste gibt es positive Anzeichen aus Frankreich, Polen und Irland. Mittel- und langfristig sollte die neue UN-Mission politisch, finanziell, materiell und personell unterstützt werden. Sowohl die Kommission als auch der Rat der Europäischen Union sollten sich stärker auf den Feldern Entmilitarisierung, Demobilisierung und Reintegration sowie Sicherheitssektorreform engagieren und vielleicht sogar in Abstimmung mit der UN eine eigene Security Sector Reform-Mission vorbereiten.

Die Europäische Union ist auf diesem Gebiet bereits seit 2005 in der Demokratischen Republik Kongo aktiv. Sie engagiert sich dort erstmals mit der ganzen Bandbreite ihres Instrumentariums auf dem afrikanischen Kontinent und hat damit dazu beigetragen, einen zentralen Krisenherd zumindest zeitweise einzudämmen. Die zurückhaltende Reaktion auf den französischen Vorschlag, eine EU-Battlegroup in den Kongo zu entsenden, um den wieder aufgeflammten Kämpfen im Osten des Landes zu begegnen, zeigt den begrenzten Willen der EU-Mitgliedstaaten, sich militärisch stärker in der Region zu engagieren. ${ }^{20}$ Die Gründe dafür sind vielfältig und reichen von mangelndem strategischen Interesse über finanzielle Erwägungen bis zu innenpolitischer Rücksichtnahme. Gleichwohl böte ein durchdachtes und international abgestimmtes Engagement im Tschad die Gelegenheit, die Brisanz des anderen großen Krisenherdes, der den Sudan, den Tschad und die Zentralafrikanische Republik umfasst, zu entschärfen. Die 2007 zwischen der Europäischen Union und der AU beschlossene strategische Partnerschaft würde durch konkretes gemeinsames Handeln in dieser Krise untermauert. Zudem entspräche ein solches Engagement nicht nur dem europäischen Interesse an einer Stabilisierung dieser Region, sondern auch dem normativen Anspruch der Europäischen Union als Friedensmacht. ${ }^{21}$

20 Vgl. Lucia Kubosova: EU resists calls to send troops to east Congo, in: EUobserver, 30.10.2008.

21 Vgl. Hans-Georg Ehrhart: Zwischen rationalistischem Kalkül und Logik der Angemessenheit: Zur Ethik des Befriedungskonzepts von VN und EU im Kongo, in: Heinz-Gerhard Justenhoven/Hans-Georg Ehrhart (Hrsg.): Intervention im Kongo. Eine kritische Analyse der Befriedungspolitik von UN und EU, Stuttgart 2008, S. 126-164. 